\title{
Direct Observation of Electrostatically Driven Band Gap Renormalization in a Degenerate Perovskite Transparent Conducting Oxide
}

\author{
Z. Lebens-Higgins, ${ }^{1}$ D. O. Scanlon, ${ }^{2, *}$ H. Paik ${ }^{3}$ S. Sallis, ${ }^{4}$ Y. Nie, ${ }^{3,5}$ M. Uchida, ${ }^{5,6}$ N. F. Quackenbush, ${ }^{1}$ \\ M. J. Wahila, ${ }^{1}$ G. E. Sterbinsky, ${ }^{7}$ Dario A. Arena, ${ }^{8}$ J. C. Woicik, ${ }^{9}$ D. G. Schlom, ${ }^{3,10}$ and L. F. J. Piper ${ }^{1,4,}$ \\ ${ }^{1}$ Department of Physics, Applied Physics and Astronomy, Binghamton University, Binghamton, 10 New York 13902, USA \\ ${ }^{2}$ Kathleen Lonsdale Materials Chemistry, Department of Chemistry, University College London, \\ 20 Gordon Street, London WC1H OAJ, United Kingdom \\ ${ }^{3}$ Department of Materials Science and Engineering, Cornell University, Ithaca, New York 14853, USA \\ ${ }^{4}$ Materials science and Engineering, Applied Physics and Astronomy, Binghamton University, \\ Binghamton, 10 New York 13902, USA \\ ${ }^{5}$ Laboratory of Atomic and Solid State Physics, Department of Physics, Cornell University, Ithaca, New York 14853, USA \\ ${ }^{6}$ Department of Applied Physics and Quantum-Phase Electronics Center (QPEC), University of Tokyo, Tokyo 113-8656, Japan \\ ${ }^{7}$ National Synchrotron Light Source, Brookhaven National Laboratory, Upton, New York 11973, USA ${ }^{\S}$ \\ ${ }^{8}$ National Synchrotron Light Source-II, Basic Energy Sciences Directorate, Brookhaven National Laboratory, \\ Upton, New York 11973, USA ${ }^{\text {『 }}$ \\ ${ }^{9}$ Materials Science and Engineering Laboratory, National Institute of Standards and Technology, \\ Gaithersburg, Maryland 20899, USA \\ ${ }^{10}$ Kavli Institute at Cornell for Nanoscale Science, Ithaca, New York 14853, United States
}

(Received 29 July 2015; revised manuscript received 21 October 2015; published 15 January 2016)

We have directly measured the band gap renormalization associated with the Moss-Burstein shift in the perovskite transparent conducting oxide (TCO), La-doped $\mathrm{BaSnO}_{3}$, using hard x-ray photoelectron spectroscopy. We determine that the band gap renormalization is almost entirely associated with the evolution of the conduction band. Our experimental results are supported by hybrid density functional theory supercell calculations. We determine that unlike conventional TCOs where interactions with the dopant orbitals are important, the band gap renormalization in $\mathrm{La}-\mathrm{BaSnO}_{3}$ is driven purely by electrostatic interactions.

DOI: 10.1103/PhysRevLett.116.027602

Transparent conducting oxides (TCOs) are typically used as passive transparent electrodes within flat panel displays and solar cell devices $[1,2]$. The realization of epitaxial TCOs by molecular beam epitaxy such as $n$-type La-doped $\mathrm{BaSnO}_{3}$ (LBSO) [3], and $p$-type $\mathrm{Sr}$-doped $\mathrm{LaCrO}_{3}[4,5]$, means that these TCOs are easily compatible with the perovskite based "oxide electronics" [6-8]. Indeed, LBSO has already been touted as an interesting alternative to $\mathrm{SrTiO}_{3}$ and its derivatives for use in oxide electronics [9]. Optimizing the electronic conductivity and optical transparency of LBSO can be achieved by the utilization of the Moss-Burstein (MB) shift [10,11], which refers to the increase in the absorption edge with degenerate doping. The MB shift is widely observed by optical measurements of III-V [12] and metal oxide semiconductors [13,14], including LBSO [15]. Despite the importance of the MB shift in tailoring the performance of TCOs, little is understood regarding the band gap renormalization that simultaneously occurs during the MB shift. LBSO, with its delocalized $s$ orbital derived conduction band minimum (CBM) [16], presents an idealized TCO system for examining the effects of band gap renormalization associated with the MB shift.

The band gap renormalization (also commonly referred to as band gap shrinkage) is typically modeled by electron-electron and electron-ion interactions in highly degenerate semiconductors [17]. Although band gap renormalization is important, there is a lack of experimental studies to verify the magnitude of these contributions. Simply put, the optical measurements used to measure the MB shift are not sensitive to the evolution of the individual band edges since they measure dipole-allowed transitions between filled and unoccupied states. As a result, parabolic band filling models are commonly used to estimate the magnitude of band gap renormalization from optical measurements. First principles calculations of degenerate doping in $\mathrm{In}_{2} \mathrm{O}_{3}$ have shown that this approximation is not adequate as the evolution of the conduction band needs to be taken into account [18]. For instance, the conduction band dispersion is significantly nonparabolic at high Fermi wave vectors and is further modified by interactions with the dopant's orbital states leading to a dependence of the band gap renormalization on the specific dopant [18]. Standard density functional theory (DFT), however, is well known for poorly determining the band gap of semiconductors [19-21]. It remains unclear whether DFT calculations can adequately consider the effects of band gap renormalization in regimes where the carrier concentrations approach experimental values, i.e., $n \sim 10^{20} \mathrm{~cm}^{-3}$. 
As a result, there is a need for direct measurements of band gap renormalization that can be explicitly compared to DFT.

Here, we employ hard x-ray photoelectron spectroscopy (HAXPES) to directly measure the band gap renormalization within LBSO as a function of La doping. Unlike optical measurements, HAXPES of degenerate semiconductors can locate the band edges on common binding energy axes and be directly compared to hybrid DFT [16]. We confirm both experimentally and computationally that the band gap renormalization observed is almost solely due to the evolution of the conduction band. We are able to rule out contributions from the dopant orbitals in LBSO that are important in conventional TCOs, such as ITO [18].

Details regarding the MBE sample growth of the $25 \mathrm{~nm}$ LBSO films on $\mathrm{TbScO}_{3}$ substrates along with the experimental settings for the transport, HAXPES, and XAS measurements are provided in the Supplemental Material [22]. $\mathrm{TbScO}_{3}(110)$ substrates were used to limit the lattice mismatch between the substrate and the epilayer, providing a smaller lattice mismatch with $\mathrm{BaSnO}_{3}$ (3.96\%) than $\mathrm{SrTiO}_{3}$ (3.905 $\AA$ or a $5.40 \%$ mismatch). Nevertheless, all the films were partially relaxed and strain-induced effects to the band edges are considered negligible; refer to the Supplemental Material [22]. Figure 1 displays the transport data of our films. Our highest doped LBSO has a carrier concentration of $n=1.65 \times 10^{20} \mathrm{~cm}^{-3}$ and a mobility of $\mu=81 \mathrm{~cm}^{2} \mathrm{~V}^{-1} \mathrm{~s}^{-1}$ at room temperature, i.e., amongst the best in the literature [40]. Details of the hybrid DFT calculations for the 5 atom atom unit cell, 40 atom $(2 \times 2 \times 2), 135$ atom $(3 \times 3 \times 3)$, and 320 atom $(4 \times 4 \times 4)$ supercells are also provided in the Supplemental Material [22].
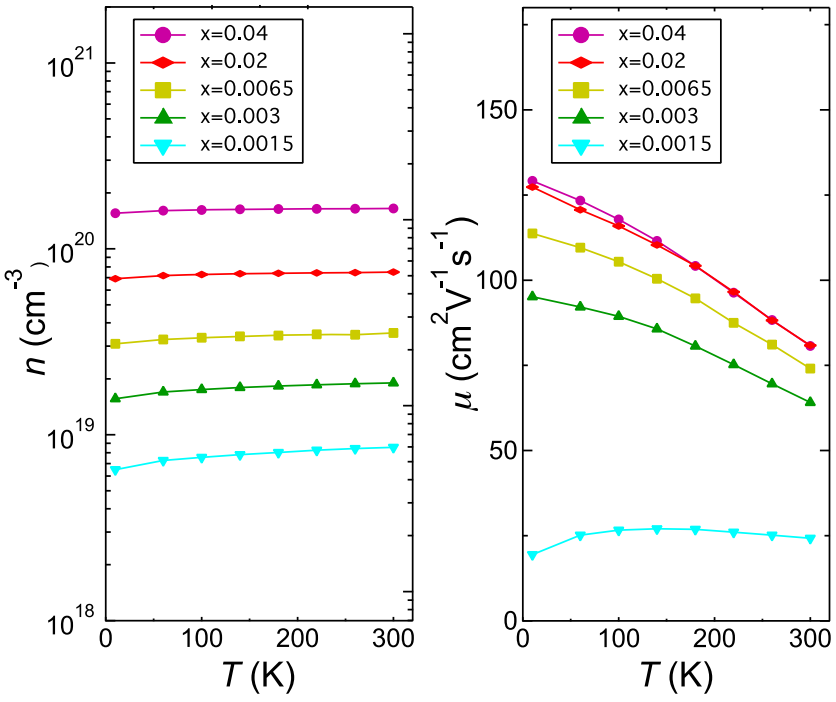

FIG. 1. Temperature dependent transport data of the $\mathrm{La}_{x} \mathrm{Ba}_{1-x} \mathrm{SnO}_{3}$ sample set displaying the carrier concentration $n$ and electron mobility $\mu$.
To correlate the CB filling in HAXPES with our transport measurements in Fig. 1, we measured the Sn 3d, La 3d, and $\mathrm{Ba} 3 d$ core levels for all the samples. Despite the reduced surface sensitivity of HAXPES, our measurements still required very low carbon coverages comparable to our noise signal to accurately observe the increase in La signal and Sn $3 d$ asymmetry with doping (refer to the Supplemental Material [22]). At $x=0.001$ $\left(\mathrm{La}_{x} \mathrm{Ba}_{1-x} \mathrm{SnO}_{3}\right)$ we observed a symmetric Sn $3 d$ line shape and could not detect any La signal. From $x=0.003$ to 0.04 , the asymmetry of the Sn $3 d$ line shape increased proportional to the La signal consistent with the measured carrier concentration.

Our HAXPES measurements were scaled to the background and aligned to the Fermi level. As the carrier concentration increases, the core levels and band edges shift to higher binding energies, as shown in Fig. 2. Conduction band filling was observed for all doping levels, even at a doping level of $x=0.001$, which was at the limit
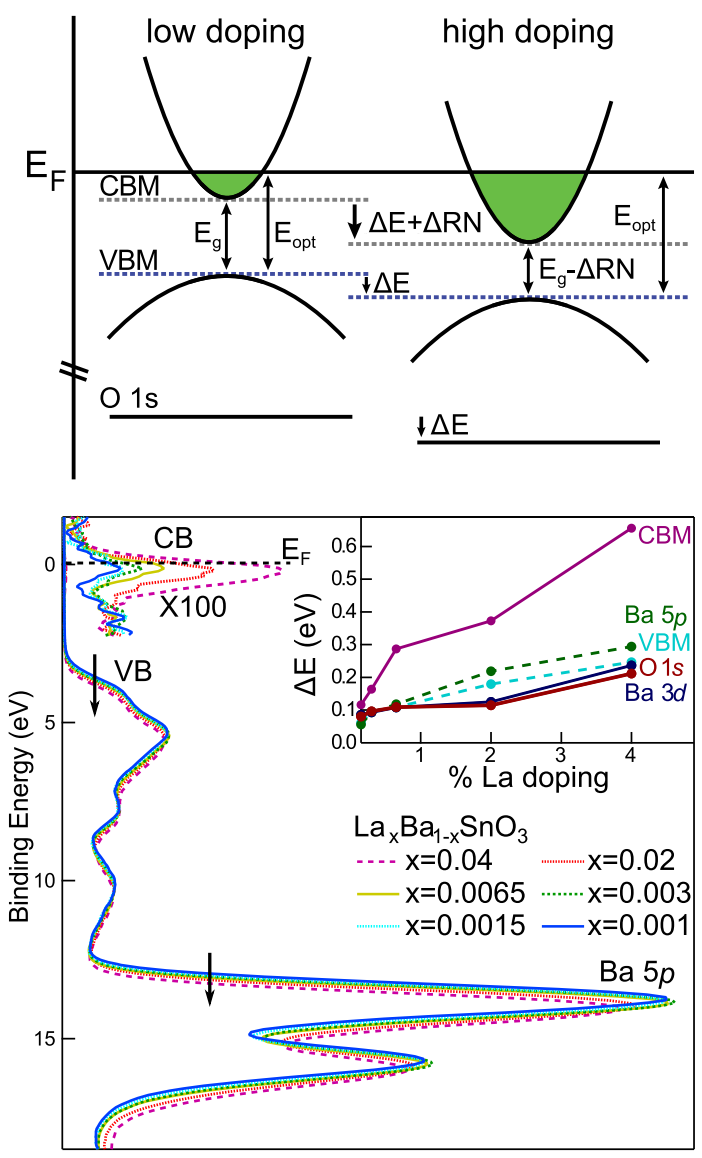

FIG. 2. (Top) A schematic of the Moss-Burstein shift observed in HAXPES. Doping shifts the orbitals with respect to the Fermi level $\left(E_{F}\right)$, increasing the optical gap $\left(E_{\mathrm{opt}}\right)$. The band gap renormalization $(\triangle \mathrm{RN})$ results from the lowering of the CBM. (Bottom) HAXPES of LBSO regions as a function of doping. The inset plots the evolution of the core levels and band edges compared to the lowest doped sample, $x=0.001$. 
of our detection. HAXPES enhances the ability to detect the $\mathrm{Sn} 5 s$ orbital compared to traditional XPS due to a more favorable photoionization cross-section ratio between the $\mathrm{O}$ $2 p$ and Sn $5 s$ orbitals $[16,41]$. Magnification of the filled Sn $5 s$ derived CB states in Fig. 2 revealed that the intensity increased simultaneously with a shift to higher binding energies upon La doping. The inset of Fig. 2 includes the energetic shift of the extrapolated conduction and valence band edges (along with the $\mathrm{Ba} 5 p$ semicore and $\mathrm{O} 1 s$ and $\mathrm{Ba} 3 d$ core levels) as a function of La doping. Details of the method used to extrapolate the band edges are included in the Supplemental Material [22]. The relative shifts were all referenced to the binding energies for the lowest doped sample, $x=0.001$. The valence band edge and core levels displayed the same shifts in binding energy with increasing doping. In contrast, the conduction band onset displayed a greater shift. We interpret these data as evidence of the band gap renormalization resulting almost entirely from the evolution of the CBM with doping, as shown schematically in Fig. 2.

The observed band filling in our HAXPES data was further examined using carrier statistics. Figure 3 displays the measured valence band maximum (VBM) with respect to the Fermi level as a function of carrier concentration. For degenerate TCOs, we note that the optical onset reflects the energetic separation of the VBM and Fermi level, i.e., the

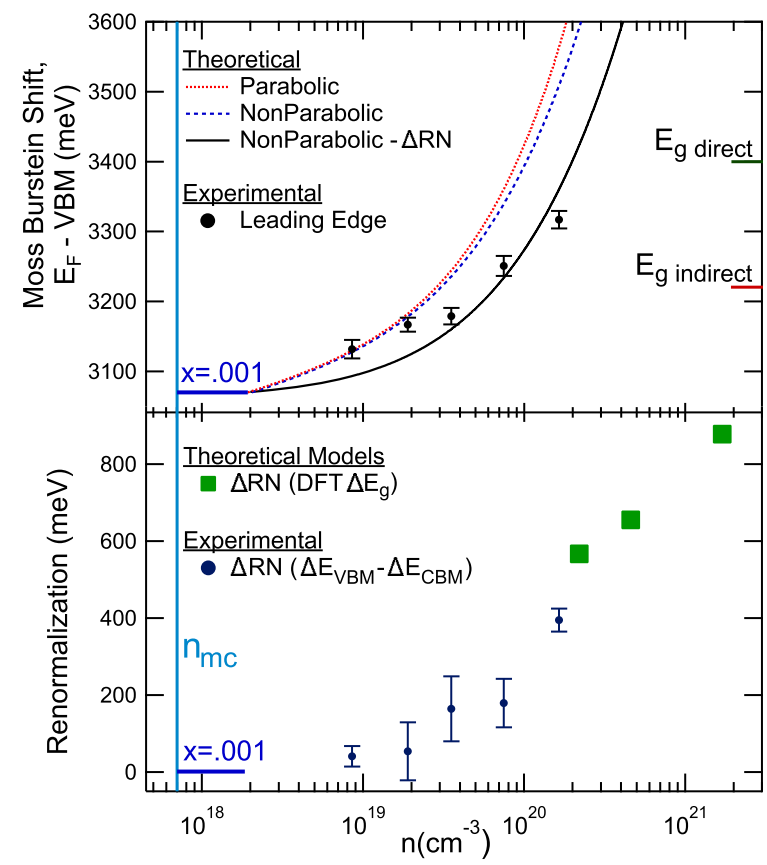

FIG. 3. (Top) The extrapolated leading edge vs carrier concentration is compared to the parabolic and nonparabolic model with and without band gap renormalization. The models were offset to the minimum leading edge of the lowest doping case, $x=0.001$. (Bottom) The amount of renormalization based on the change in the extrapolated band gap and DFT calculations. The error bars reflect the variance in extrapolating the band edges.
MB shift. We have modeled the carrier statistics using parabolic and nonparabolic models with and without band gap renormalization effects for comparison. The nonparabolic conduction band dispersion due to the interaction between the conduction band and the valence band can be approximated by

$$
E_{C}(k)\left(1+\frac{E_{C}(k)}{E_{g}}\right)=\frac{\hbar^{2} k^{2}}{2 m_{0}^{*}} .
$$

Equation (1) is solely dependent on two parameters that can, in principle, be determined experimentally, i.e., the band gap $E_{g}$ and conduction band edge effective mass $m_{0}^{*}$. Furthermore, it is also physically intuitive, with the size of the band gap largely determining the amount of deviation from the parabolic description. Here, we used an $E_{g}$ of $3.4 \mathrm{eV}$ and $m_{0}^{*}$ of $0.22 m_{0}$ [16,42]. For the range of carrier concentrations of interest, there is limited variation between the parabolic and nonparabolic model. For the renormalization component $(\Delta \mathrm{RN})$, we have applied electronelectron interactions $\Delta E^{e e}$ and electron-ion interactions $\Delta E^{e i}$ [17], such that

$$
\Delta E_{g}^{e e}=-\frac{2 e^{2} k_{F}}{\pi \epsilon_{s}}-\frac{e^{2} k_{\mathrm{TF}}}{2 \epsilon_{s}}\left(1-\frac{4}{\pi} \arctan \left(\frac{k_{F}}{k_{\mathrm{TF}}}\right)\right),
$$

where the Fermi wave vector is given by the relation $k_{F}^{3}=3 \pi^{2} n, k_{\mathrm{TF}}=2 / \sqrt{\pi}\left(k_{F} / a_{0}\right)$ is the Thomas-Fermi screening wave vector, where $a_{0}=\epsilon_{s} \hbar^{2} / m_{F}^{*} e^{2}$ is the effective Bohr radius, $m_{F}^{*}$ is the effective mass at the Fermi level in the conduction band, $\epsilon_{s}$ is the static dielectric constant, and

$$
\Delta E_{g}^{e i}=-\frac{4 \pi n e^{2}}{\epsilon_{s} a_{0} k_{\mathrm{TF}}^{3}}
$$

We have employed $\epsilon_{s}=22.3$ [43]. We note that this model was successfully applied for studying the large MB shift in InN [12]. Offsetting the models to the leading edge of the lowest doped sample, $x=0.001$, at $3.11 \mathrm{eV}$ accounts for the difference between the direct and indirect band gap. The lowest doped sample was unmeasurable with Hall measurements indicating a very low carrier concentration, such that we can approximate its measured VBM to $E_{F}$ separation as the band gap. We find that our measured $\mathrm{MB}$ shift from HAXPES agrees well with the nonparabolic model that includes only electron-electron and electron-ion contributions, as shown in Fig. 3.

The evolution of the band structure of $\mathrm{BaSnO}_{3}$ upon $\mathrm{La}$ doping was further examined. Figure 4 displays the calculated band structures for select doping limits. These calculations support our experimental results, with the band gap renormalization resulting almost entirely from the evolution of the conduction band (referenced to the $\mathrm{O} 1 \mathrm{~s}$ core level). We plot the calculated and measured 
(a) Pure

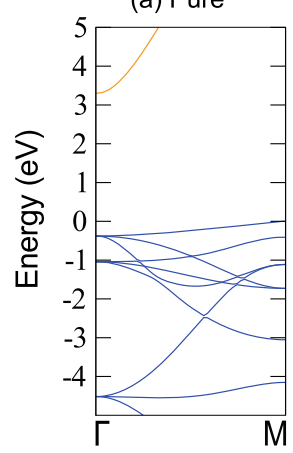

(b) $2.2 \times 10^{20} \mathrm{~cm}^{-3}$

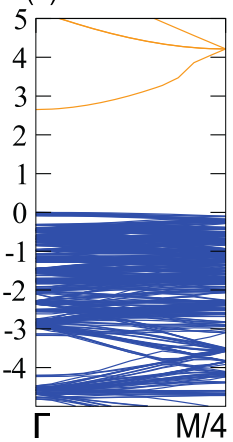

(c) $4.6 \times 10^{20} \mathrm{~cm}^{-3}$

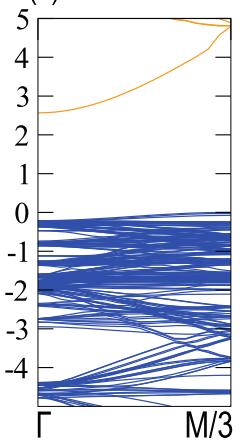

(d) $1.7 \times 10^{21} \mathrm{~cm}^{-3}$

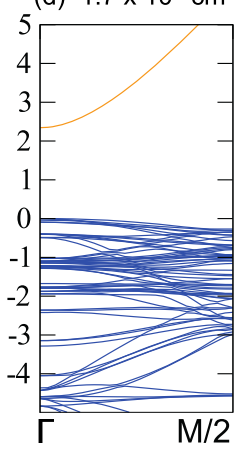

(e)

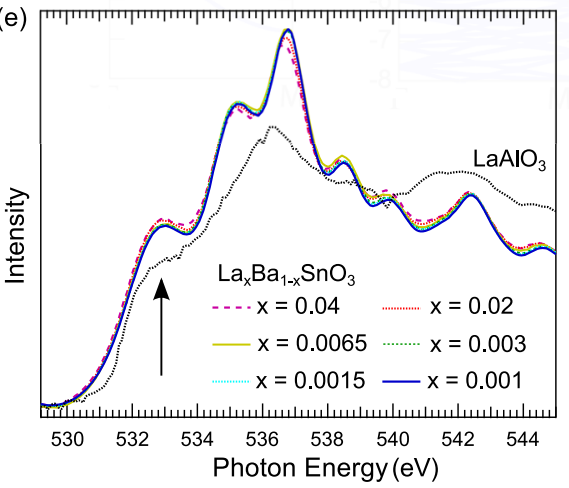

(f)

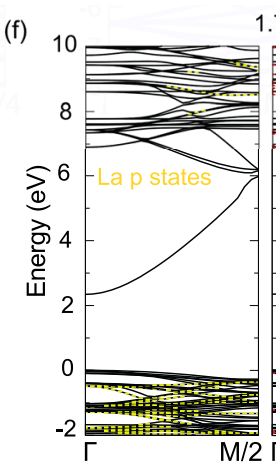

$1.7 \times 10^{21} \mathrm{~cm}^{-3}$

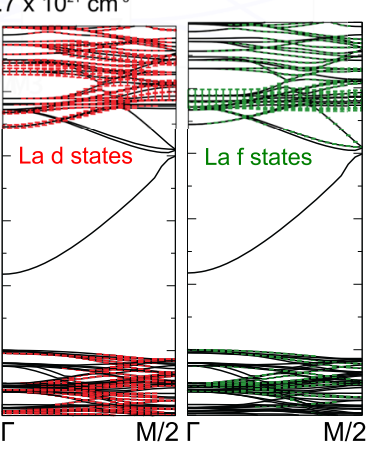

FIG. 4. (Top) PBE0 calculated band structures for (a) pure $\mathrm{BaSnO}_{3}$, (b) 1 dopant in a 320 atom super cell, (c) 1 dopant in a 135 atom super cell, and (d) 1 dopant in a 40 atom super cell. (e) The O $K$-edge XAS of LBSO and reference $\mathrm{LaAlO}_{3}$. The arrow highlights increased La-O orbital weight with La doping, high above the CBM. (f) The fat band analysis of the La orbital character of the calculated La-doped $\mathrm{BaSnO}_{3}$ band structure, the colored bars reflect the orbital weight of the bands. conduction band reduction with doping in Fig. 3. The experimental band gap renormalization refers to the measured difference between the shifts of the extrapolated CB and VB edges in HAXPES, as shown in Fig. 2. It is clear that the calculated band gap shrinkage is comparable to the observed renormalization in our experiments.

Figure 4(e) plots of the O $K$-edge XAS of our LBSO samples. We have previously shown that the $\mathrm{O} K$-edge XAS spectra can be reproduced by the calculated $\mathrm{O} 2 p$ partial density of states from hybrid DFT [16]. From inspection of the $\mathrm{O} K$-edge XAS of our La-doped $\mathrm{BaSnO}_{3}$, along with comparisons to $\mathrm{LaAlO}_{3}$, we determine the La-O hybridized states to be at least $3 \mathrm{eV}$ above the CBM of LBSO; refer to the Supplemental Material [22]. This is confirmed by fat band analysis (i.e., La orbital weighting of the band) of our hybrid DFT supercell calculations, where the La hybridized states lie at least $4 \mathrm{eV}$ above the CBM of LBSO in Fig. 4(f). As a result, the band gap renormalization of LBSO is not further modified by additional hybridization effects. This is in contrast to traditional $n$-type TCOs, for example, the conduction band of $\mathrm{In}_{2} \mathrm{O}_{3}$ is significantly modified due to dopant $\mathrm{Sn} 5 s$ and Ge $4 s$ orbital contributions lying less than $1 \mathrm{eV}$ above the CBM [18].

In conclusion, we examined the nature of the band gap renormalization associated with the Moss-Burstein shift in the La-doped $\mathrm{BaSnO}_{3}$. We demonstrate that the band gap renormalization of a TCO results from the modification of the conduction band with doping. In the case of La-doped $\mathrm{BaSnO}_{3}$, the additional modifications in the conduction band due to interactions with the dopant orbitals are negligible. As a result, our studies reveal the minimum extent of band gap renormalization in TCOs since $\mathrm{La}-\mathrm{BaSnO}_{3}$ represents an idealized case where the renormalization is driven solely by electrostatic effects. This unique property is attributed to the lack of significant orbital mixing between the CBM and dopant states in $\mathrm{La}-\mathrm{BaSnO}_{3}$. These data reaffirm the promise of $\mathrm{La}-\mathrm{BaSnO}_{3}$ as an ideal TCO in terms of band structure. The ability to dope on the $A$ site that does not significantly contribute to the orbital character of the conduction band provides perovskite TCOs an advantage over conventional TCOs. Quite simply, one would expect to be able to achieve even higher figures of merit regarding the optical transparency and electrical conductivity with epitaxial films of La-doped $\mathrm{BaSnO}_{3}$ by minimizing the lattice mismatch between the thin film and the substrate to further strain engineer $\mathrm{BaSnO}_{3}$.

We thank A. Sokol and K. Shen for discussions. The research at Binghamton was partially supported by the National Science Foundation under Grant No. DMR1409912. Z. W. L.-H. is grateful for an undergraduate Summer Scholars and Artists Award from Binghamton University. D. O.S acknowledges access to the ARCHER supercomputer via membership of the UK's HPC Materials Chemistry Consortium, which is funded by EPSRC Grants No. EP/L000202, and No. EP/N01572X/1. The work of H. P. and D. G. S. was supported by the Center for Low Energy Systems Technology (LEAST), one of six centers of STARnet, a Semiconductor Research Corporation program sponsored by MARCO and DARPA. This work made use of the Cornell Center for Materials Research Shared 
Facilities, which are supported through the NSF MRSEC Program (Grant No. DMR-1120296). This work was performed in part at the Cornell Nanoscale Facility, a member of the National Nanotechnology Infrastructure Network, which is supported by the National Science Foundation (Grant No. ECCS-0335765) The NSLS is supported by the U.S. Department of Energy, Office of Science, Office of Basic Energy Sciences, under Contract No. DE-AC02-98CH10886. Beam line X24a at the NSLS is supported by the National Institute of Standards and Technology.

*d.scanlon@ucl.ac.uk

†piper@binghamton.edu

Present address: National Laboratory of Solid State Microstructures and College of Engineering and Applied Sciences, Nanjing University, Nanjing, 210093, People's Republic of China.

${ }^{\S}$ Present address: Advanced Photon Source, Argonne National Laboratory, Argonne, Illinois 60439, USA.

"Present address: Department of Physics, University of South Florida, Tampa, Florida 33620, USA.

[1] D. S. Ginley and C. Bright, MRS Bull. 25, 15 (2000).

[2] E. Fortunato, D. Ginley, H. Hosono, and D. C. Paine, MRS Bull. 32, 242 (2007).

[3] U. Kim, C. Park, T. Ha, Y. M. Kim, N. Kim, C. Ju, J. Park, J. Yu, J. H. Kim, and K. Char, APL Mater. 3, 036101 (2015).

[4] K. H. L. Zhang, Y. Du, P. V. Sushko, M. E. Bowden, V. Shutthanandan, S. Sallis, L. F. J. Piper, and S. A. Chambers, Phys. Rev. B 91, 155129 (2015).

[5] K. H. L. Zhang, Y. Du, A. Papadogianni, O. Bierwagen, S. Sallis, L. F. J. Piper, M. E. Bowden, V. Shutthanandan, P. V. Sushko, and S. A. Chambers, Adv. Mater. 27, 5191 (2015).

[6] A. Ohtomo and H. Y. Hwang, Nature (London) 427, 423 (2004).

[7] D. G. Schlom, L.-Q. Chen, C.-B. Eom, K. M. Rabe, S. K. Streiffer, and J.-M. Triscone, Annu. Rev. Mater. Res. 37, 589 (2007).

[8] J. Mannhart and D. G. Schlom, Science 327, 1607 (2010).

[9] S. Ismail-Beigi, F. J. Walker, S.-W. Cheong, K. M. Rabe, and C. H. Ahn, APL Mater. 3, 062510 (2015).

[10] T. S. Moss, Proc. Phys. Soc. London Sect. B 67, 775 (1954).

[11] E. Burstein, Phys. Rev. 93, 632 (1954).

[12] J. Wu, W. Walukiewicz, W. Shan, K. M. Yu, J. W. Ager, E. E. Haller, H. Lu, and W. J. Schaff, Phys. Rev. B 66, 201403 (2002).

[13] S. Parthiban, E. Elangovan, K. Ramamurthi, R. Martins, and E. Fortunato, J. Appl. Phys. 106, 063716 (2009).

[14] P. Banerjee, W.-J. Lee, K.-R. Bae, S. B. Lee, and G. W. Rubloff, J. Appl. Phys. 108, 043504 (2010).

[15] D. Seo, K. Yu, Y. Jun Chang, E. Sohn, K. Hoon Kim, and E. J. Choi, Appl. Phys. Lett. 104, 022102 (2014).

[16] S. Sallis, D. O. Scanlon, S. C. Chae, N. F. Quackenbush, D. A. Fischer, J. C. Woicik, J.-H. Guo, S. W. Cheong, and L. F. J. Piper, Appl. Phys. Lett. 103, 042105 (2013).
[17] K.-F. Berggren and B. E. Sernelius, Phys. Rev. B 24, 1971 (1981).

[18] A. Walsh, J. L. F. Da Silva, and S.-H. Wei, Phys. Rev. B 78, 075211 (2008).

[19] A. K. Singh, A. Janotti, M. Scheffler, and C. G. Van de Walle, Phys. Rev. Lett. 101, 055502 (2008).

[20] M. Burbano, D. O. Scanlon, and G. W. Watson, J. Am. Chem. Soc. 133, 15065 (2011).

[21] P. Ágoston, K. Albe, R. M. Nieminen, and M. J. Puska, Phys. Rev. Lett. 103, 245501 (2009).

[22] See Supplemental Material at http://link.aps.org/ supplemental/10.1103/PhysRevLett.116.027602 for experimental and computational details, XPS of core regions, and XAS of O K-edge and $\mathrm{Sn} \mathrm{M}_{4,5}$ edge, which includes Refs. [23-39].

[23] K. Gunther, Naturwissenschaften 45, 415 (1958).

[24] C. Theis, J. Yeh, D. Schlom, M. Hawley, and G. Brown, Thin Solid Films 325, 107 (1998).

[25] J. F. Ihlefeld, N. J. Podraza, Z. K. Liu, R. C. Rai, X. Xu, T. Heeg, Y. B. Chen, J. Li, R. W. Collins, J. L. Musfeldt et al., Appl. Phys. Lett. 92, 142908 (2008).

[26] R. E. Honig and D. A. Kramer, RCA Rev. 30, 285 (1969).

[27] G. Kresse and J. Furthmüller, Phys. Rev. B 54, 11169 (1996).

[28] G. Kresse and D. Joubert, Phys. Rev. B 59, 1758 (1999).

[29] J. P. Perdew, K. Burke, and M. Ernzerhof, Phys. Rev. Lett. 77, 3865 (1996).

[30] C. Adamo and V. Barone, J. Chem. Phys. 110, 6158 (1999).

[31] P. Agoston, C. Korber, A. Klein, M. J. Puska, R. M. Nieminen, and K. Albe, J. Appl. Phys. 108, 053511 (2010).

[32] D. O. Scanlon and G. W. Watson, J. Mater. Chem. 22, 25236 (2012).

[33] C. Körber, V. Krishnakumar, A. Klein, G. Panaccione, P. Torelli, A. Walsh, J. L. F. Da Silva, S.-H. Wei, R. G. Egdell, and D. J. Payne, Phys. Rev. B 81, 165207 (2010).

[34] J. Jia, N. Oka, and Y. Shigesato, J. Appl. Phys. 113, 163702 (2013).

[35] J. N. Chazalviel, M. Campagna, G. K. Wertheim, and H. R. Shanks, Phys. Rev. B 16, 697 (1977).

[36] J. J. Mudd, T.-L. Lee, V. Muñoz-Sanjosé, J. Zúñiga-Pérez, D. Hesp, J. M. Kahk, D. J. Payne, R. G. Egdell, and C. F. McConville, Phys. Rev. B 89, 035203 (2014).

[37] S. Sallis, L. F. J. Piper, J. Francis, J. Tate, H. Hiramatsu, T. Kamiya, and H. Hosono, Phys. Rev. B 85, 085207 (2012).

[38] S. O. Kucheyev, T. F. Baumann, P. A. Sterne, Y. M. Wang, T. van Buuren, A. V. Hamza, L. J. Terminello, and T. M. Willey, Phys. Rev. B 72, 035404 (2005).

[39] M. S. Moreno, R. F. Egerton, and P. A. Midgley, Phys. Rev. B 69, 233304 (2004).

[40] H. J. Kim, U. Kim, H. M. Kim, T. H. Kim, H. S. Mun, B.-G. Jeon, K. T. Hong, W.-J. Lee, C. Ju, K. H. Kim et al., Appl. Phys. Express 5, 061102 (2012).

[41] N. F. Quackenbush, J. P. Allen, D. O. Scanlon, S. Sallis, J. A. Hewlett, A. S. Nandur, B. Chen, K. E. Smith, C. Weiland, D. A. Fischer et al., Chem. Mater. 25, 3114 (2013).

[42] D. O. Scanlon, Phys. Rev. B 87, 161201 (2013).

[43] E. Bévillon, A. Chesnaud, Y. Wang, G. Dezanneau, and G. Geneste, J. Phys. Condens. Matter 20, 145217 (2008). 